\title{
INERTIAL MEASUREMENT UNIT ERROR REDUCTION BY CALibration USING DifFerential Evolution
}

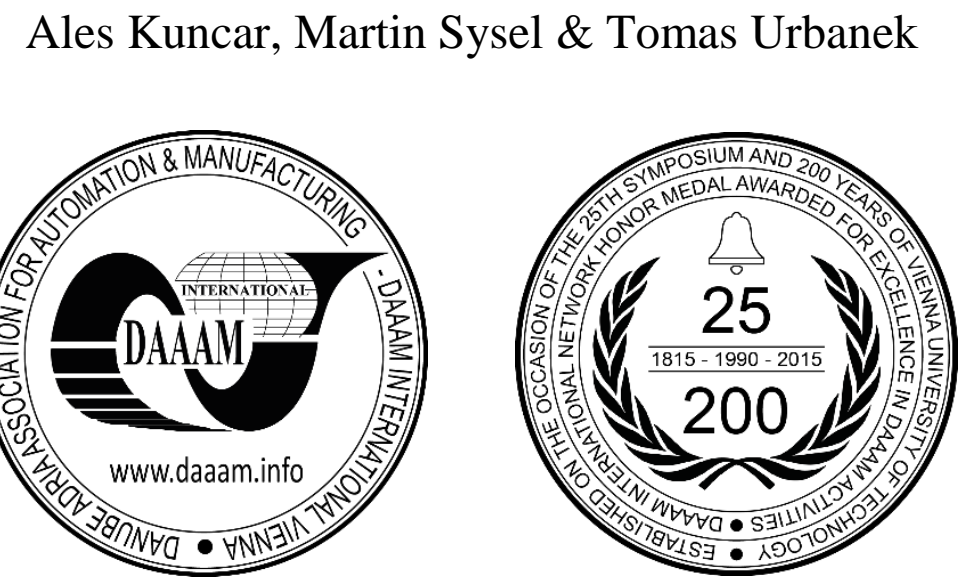

This Publication has to be referred as: Kuncar, A[les]; Sysel, M[artin] \& Urbanek, T[omas] (2018). Inertial Measurement Unit Error Reduction by Calibration Using Differential Evolution, Proceedings of the 29th DAAAM International Symposium, pp.0681-0686, B. Katalinic (Ed.), Published by DAAAM International, ISBN 978-3-90273420-4, ISSN 1726-9679, Vienna, Austria

DOI: $10.2507 / 29$ th.daaam.proceedings.098

\begin{abstract}
The sensors, such as accelerometer, gyroscopes, and magnetometers, based on the MEMS (Micro-Electro-Mechanical System) technology are widely used in various engineering areas and applications. The main advantages of these sensors are its size, price and power consumption; however, measurements of the sensors are not entirely accurate. They are influenced by sensor errors (scale factor, and bias), manufacturing errors (misalignment), and environmental errors (magnetic deviations). Therefore, the error reduction using calibration is a crucial and actual problem. In this research paper, we have presented a calibration method using differential evolution to determine the correction parameters. The performance of this method was tested on 9DOF (degree of freedom) IMU STEVAL-MKI124V1 which consists of three axis accelerometer and three axis magnetometer (LSM303DLHC) and gyroscope (L3GD20).
\end{abstract}

Keywords: accelerometer; calibration; differential evolution; gyroscope; magnetometer

\section{Introduction}

Recently, the Micro-Electro-Mechanical System (MEMS) technology has an impact in many engineering areas and applications. These technologies are widely used in various areas such as information technology [1][2], automotive industry, military defence [3][4], and inertial localization and navigation systems [5][6].

These low-cost sensors (accelerometers, gyroscopes, and magnetometers) are small, cheap, and have low power consumption. On the other hand, the precision is affected by systematic errors (scale factor, bias, misalignment angles, temperature, etc.). Therefore, the proper calibration must be provided to reduce error to improve the accuracy of sensor measurements. Calibration is a technique or procedure of measuring of known information and then estimating the calibration parameters to correct sensor readings. Many sensors are factory calibrated even though this calibration is insufficient.

In this research paper, we proposed a calibration method for 9DOF (degree of freedom) IMU (inertial measurement unit) which consists of three axis accelerometer, three axis gyroscope, and three axis magnetometer. This method uses differential evolution which is described in [7]. 
In present, many researchers deal with the calibration of low-cost inertial sensors based on MEMS technology. The traditional methods for calibration of the IMU are to calibrate each sensor separately. In [8], Frosio et al. presented onfield calibration for three axis accelerometer which does not require any special equipment. This procedure minimizes the bias, scale factor and cross axis errors. For magnetometers, Guo et al. [9] and Crassidis et al. [10] used Kalman filters to compensate magnetic interferences and sensor errors.

On the other hand, Ammann et al. [11] presented the calibration method for three axis accelerometer and magnetometer based on least square method. Olsson et al. [12] used maximum likelihood on accelerometer and gyroscope. In [13], Thang et al. calibrated 9DOF IMU using rotation platform. This method not only compensates the scale factor and bias, but also covers the correction of angle correlations between sensors. The reminder of this paper is organized as follows. The sources of error are briefly described in section 2 . We have focused on the sensor error model in section 3 and finally, the IMU calibration and its details are mentioned in section 4.

\section{Sources of Error}

The MEMS sensors are influenced by many sources of errors such as sensor errors (scale factor, zero deviation), installation errors (misalignment), and environmental errors (magnetic interferences).

\subsection{Scale Factor Error}

Scale factor error or sensitivity error is a proportional relation between the output error (deviation from the straight line) and input. The scale factor can be modelled as:

$$
\boldsymbol{S}=\operatorname{diag}\left(\begin{array}{lll}
s_{X} & s_{Y} & s_{Z}
\end{array}\right)
$$

\subsection{Misalignment Error}

The misalignment error is caused by the imperfection during the installation of the sensors on the PCB (printed circuit board). This error is defined as the angles between the sensor axis and the device body axis, and it is represented as:

$$
\boldsymbol{M}_{\boldsymbol{m}}=\left[\begin{array}{lll}
1 & 0 & 0 \\
\alpha & 1 & 0 \\
\beta & \gamma & 1
\end{array}\right]
$$

\subsection{Zero Deviation}

Even when the sensor is not exposed to any movement or magnetic field, the output is non-zero value. This is caused by the small voltage in the sensor. This error can be expressed as:

$$
\boldsymbol{O}=\left[\begin{array}{lll}
o_{X} & o_{Y} & o_{Z}
\end{array}\right]^{T}
$$

\subsection{Magnetic Interferences}

The magnetic interferences can be divided up into two groups: soft-iron and hard-iron interference. The hard-iron interference is caused by the presence of magnets or materials generating fixed or slightly time-varying magnetic field.

$$
\boldsymbol{S} \boldsymbol{I}=\left[\begin{array}{lll}
s i_{X X} & s i_{X Y} & s i_{X Z} \\
s i_{Y X} & s i_{Y Y} & s i_{Y Z} \\
s i_{Z X} & s i_{Z Y} & s i_{Z Z}
\end{array}\right]
$$

The second type, soft-iron interference, occurs when a ferromagnetic material is in the vicinity of the sensor or it can be even generated by the device itself.

$$
\boldsymbol{H}=\left[\begin{array}{lll}
h_{X} & h_{Y} & h_{Z}
\end{array}\right]^{T}
$$

\section{Sensor Error Model}

\subsection{Accelerometer}

The accelerometers measure the constant gravity in static position or acceleration/vibration of an object. However, these measurements are influenced by axis misalignment, scale factor errors, zero deviation, and wide-band noise. These errors in accelerations lead to continual grow of the error in velocity and distance, due to integration. 
The mathematical model, which describes the accelerometer readings, is expressed by

$$
\vec{a}=\boldsymbol{M}_{\boldsymbol{m}} \cdot \boldsymbol{S} \cdot(\vec{M}+\boldsymbol{O}+\boldsymbol{n})
$$

where the variables $\boldsymbol{M}_{\boldsymbol{m}}$, and $\boldsymbol{S}$ are $3 \times 3$ matrices which interpret misalignment errors, and scale factors, respectively. $\boldsymbol{O}$ and $\boldsymbol{n}$ are vectors representing zero deviation and wide-band noise (Gaussian noise) which influences the acceleration vector $\vec{M}$ in the local reference system.

\subsection{Gyroscope}

The gyroscopes measure angular velocity (rotations in general) around each sensitive axis. For these measurements $\vec{M}$, the model is similar to accelerometer error model. It includes misalignment error $\boldsymbol{M}_{\boldsymbol{m}}$, scale factor error $\boldsymbol{S}$, zero deviation $\boldsymbol{O}$, and wide-band noise $\boldsymbol{n}$.

Mathematical model, that describes the measurements of the gyroscope, is expressed

$$
\vec{g}=M_{m} \cdot S \cdot(\vec{M}+\boldsymbol{O}+\boldsymbol{n})
$$

\subsection{Magnetometer}

The strength and direction of the local magnetic field is measured by the magnetometers. The measured magnetic field is a combination of the Earth's magnetic field and a magnetic noise which is generated by nearby objects or even the device itself. The magnetic noise is called soft iron $\boldsymbol{S I}$ and hard iron $\boldsymbol{H}$.

The magnetometer error model is represented as,

$$
\vec{m}=\boldsymbol{M}_{\boldsymbol{m}} \cdot \boldsymbol{S} \cdot \boldsymbol{S I} \cdot(\vec{M}+\boldsymbol{H}+\boldsymbol{n})
$$

In this model, the variables $\boldsymbol{M}_{\boldsymbol{m}}, \boldsymbol{S}$ and $\boldsymbol{S} \boldsymbol{I}$ are 3x3 matrices which represent misalignment errors, scale factors and soft-iron, respectively. $\boldsymbol{H}$ and $\boldsymbol{n}$ are vectors representing hard-iron biases and wideband noise which distorts the true magnetic field measurements $\vec{M}$.

\section{Calibration}

For the purpose of estimation of calibration parameters, the differential evolution was used due to its optimization technique. The fitness function was different for each sensor. This modelled fitness functions depend on the number of unknown parameters and attributes of the sensor. However, the goal was the same; finding the smallest value of the error.

\subsection{Three Axis Accelerometer}

For the calibration of three axis accelerometer, measurements in several different static positions for the determination of scale factor, misalignment angles and offset is required. The measured dataset consists of 12 different positions for each sensitive axis. The readings were provided for $30^{\circ}$ until the full circle is covered. The sum of squared errors has been used as the fitness function where the error is the difference between the calculated output from current parameters and the true value. Therefore, the fitness function of the DE algorithm is modelled as

$$
F=\sum_{i=1}^{n}\left(\sqrt{X^{2}+Y^{2}+Z^{2}}-1\right)^{2}
$$

where $X, Y$, and $Z$ are calibrated values and value 1 is the gravitation field given in $g$-force acceleration $(1 g=9.809228$ $\left.m \cdot s^{-2}\right)$.

The calibrated values account for bias offset $\left(o_{X}, o_{Y}, o_{Z}\right)$, scale factor $\left(s_{X}, s_{Y}, s_{Z}\right)$, and misalignment error $((\alpha, \beta, \gamma))$. The measured values are $r_{X}, r_{Y}$ and $r_{Z}$. The equations for calculations of such errors are showed in (10), (11), (12).

$$
\begin{aligned}
& X=\left(r_{X}-o_{X}\right) \cdot s_{X} \\
& Y=\left(r_{X}-o_{X}\right) \cdot \alpha+\left(r_{Y}-o_{Y}\right) \cdot s_{Y} \\
& Z=\left(r_{X}-o_{X}\right) \cdot \beta+\left(r_{Y}-o_{Y}\right) \cdot \gamma+\left(r_{Z}-o_{Z}\right) \cdot s_{Z}
\end{aligned}
$$




\subsection{Three Axis Gyroscope}

To reduce the errors, especially systematic errors (scale factor, bias), several measurements are needed in known conditions. In our case, we had used simple turntable and measure angular velocity in each sensitive axis. The fitness function is based on the sum of the squared errors where the error is the difference between the calculated output from current parameters and the true value. The fitness function is modelled as

$$
F=\sum_{i=1}^{n}(X+Y+Z-\Omega)^{2}
$$

where $X, Y$, and $Z$ are calibrated values and $\Omega$ is the known angular velocity (198 dps). The $X, Y$, and $Z$ are similar to accelerometer (10), (11), (12).

\subsection{Three Axis Magnetometer}

In order to determine the calibration parameters of the magnetometer, sufficient number of samples is needed in different directions to cover the sphere. Therefore the data were measured while rotating the sensor around each sensitive axis and also in arbitrary rotations. Duplicate readings were deleted from the dataset to avoid influencing calculation of parameters. Again, the fitness function is the sum of squared errors where the error is the difference between the calculated output from current parameters and the true value. The fitness function is represented as

$$
F=\sum_{i=1}^{n}\left(\sqrt{X^{2}+Y^{2}+Z^{2}}-R\right)^{2}
$$

where $X, Y$, and $Z$ are calibrated values in each axis and $R$ is the true scalar value of geomagnetic field intensity taken from IGRF [14] due to the absence of proton magnetometer.

The equations for the calculation of the fitness function are

$$
\begin{aligned}
& X=\left(r_{X}-o_{X}\right) \cdot s_{X}+\left(r_{Y}-o_{Y}\right) \cdot s i_{X Y} \cdot s_{X}+\left(r_{Z}-o_{Z}\right) \cdot s i_{X Z} \cdot s_{X} \\
& Y=\left(r_{X}-o_{X}\right) \cdot\left(\alpha \cdot s_{X}+s i_{Y X} \cdot s_{Y}\right)+\left(r_{Y}-o_{Y}\right) \cdot\left(\alpha \cdot s i_{X Y} \cdot s_{X}+s_{Y}\right)+\left(r_{Z}-o_{Z}\right) \cdot\left(\alpha \cdot s i_{X Z} \cdot s_{X}+s i_{Y Z} \cdot s_{Y}\right) \\
& \begin{aligned}
Z=\left(r_{X}-o_{X}\right) \cdot\left(\beta \cdot s_{X}+\gamma \cdot s i_{Y X} \cdot s_{Y}+s i_{Z X} \cdot s_{Z}\right)+\left(r_{Y}-o_{Y}\right) \cdot\left(\beta \cdot s i_{X Y} \cdot s_{X}+\gamma \cdot s_{Y}+s i_{Z Y} \cdot s_{Z}\right) \\
\quad+\left(r_{Z}-o_{Z}\right) \cdot\left(\beta \cdot s i_{X Z} \cdot s_{X}+\gamma \cdot s i_{Y Z} \cdot s_{Y}+s_{Z}\right)
\end{aligned}
\end{aligned}
$$

\subsection{Evaluation of Calibration Method}

For the evaluation of the calibration technique, data was analyzed. We used RMSE (root mean square error) as a performance criterion. This criterion indicates how accurate the calibration method is; therefore, the lower the RMSE value is the more precise are measurements. These values for each sensor are depicted in Table 1.

\begin{tabular}{|c|c|c|}
\hline & RMSE raw & RMSE calibrated \\
\hline Accelerometer & 0.0304 & 0.0082 \\
\hline Gyroscope & 1.2437 & 0.2886 \\
\hline Magnetometer & 128.6846 & 8.2388 \\
\hline
\end{tabular}

Table 1. Comparison of RMSE of each sensor before and after calibration

From results of RMSE analysis, it is clear that differential evolution is suitable as a 9DOF IMU calibration tool. This method significantly increases the precision of the magnetometer in particular, in which the measurement error was caused by the metal housing of the measuring unit. This housing caused an offset (hard-iron interference). As second analysis, we used descriptive statistics (see Table 2). This statistical analysis showed that there was a reduction in standard deviation (SD) which increases the precision of each sensor.

\begin{tabular}{|c|c|c|c|c|c|}
\hline & Mean & SD & Median & Min & Max \\
\hline AccRaw & 1 & 0.03 & 1 & 0.89 & 1.07 \\
\hline AccDE & 1 & 0.01 & 1 & 0.95 & 1.03 \\
\hline GyroRaw & 198.81 & 0.94 & 198.81 & 195.25 & 202.28 \\
\hline GyroDE & 198.01 & 0.29 & 198.01 & 196.29 & 199.29 \\
\hline MagRaw & 487.02 & 128.66 & 532.95 & 189.84 & 707.43 \\
\hline MagDE & 490.27 & 8.23 & 491.53 & 463.91 & 511.44 \\
\hline
\end{tabular}

Table 2. Descriptive statistics of raw and calibrated measurements 


\subsection{Setup of Differential Evolution}

The set-up of differential evolution is shown in Table 3. The parameter $N P$ is set to the highest value of the limitation suggested by [15]. That means $10 \cdot D$ where $D$ represents the dimension of the problem (the number of unknown calibration parameters). The number of generations is given by the experiment that showed the optimal value around 400 (slow convergence rate to minimum - see Fig. 1. The parameters $F$ and $C R$ were also determined empirically.

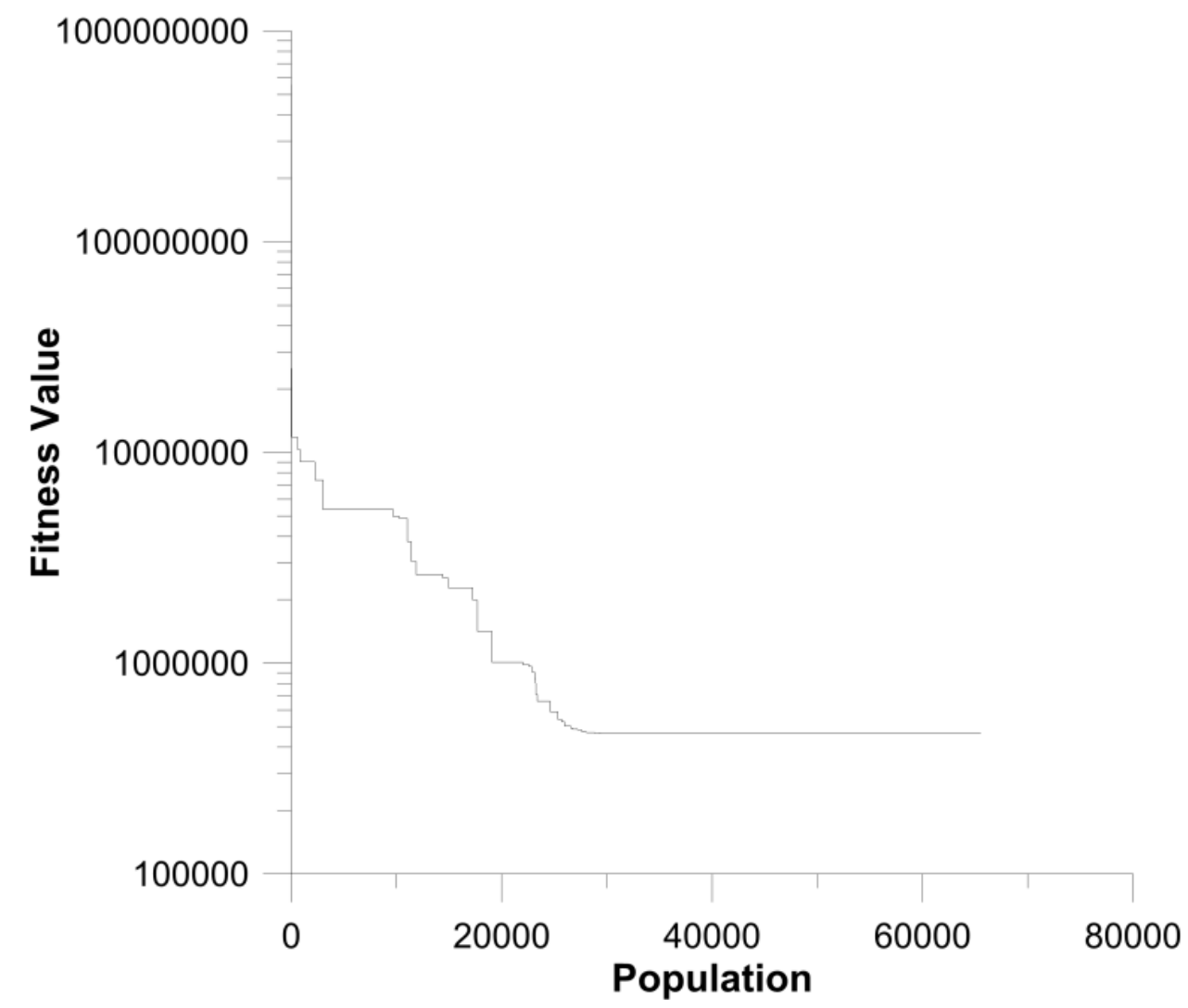

Fig. 1. Fitness function evolution

\begin{tabular}{|c|c|}
\hline Parameters & Value \\
\hline NP & 90 \\
\hline Generations & 400 \\
\hline F & 0.4 \\
\hline CR & 0.6 \\
\hline
\end{tabular}

Table 3. Set-up of differential evolution

\section{Conclusion}

In this research paper, we presented a calibration method for 9DOF inertial measurement unit. This calibration method uses differential evolution as a optimization technique for determination of calibration parameters. This method compensates misalignment, scale factor, bias and magnetic deviations. The accuracy of calibration depends on the amount of measured data. The calibration was tested on accelerometer and magnetometer (LSM303DLHC), and gyroscope (L3GD20) from STMicroelectronics. The experiment showed a significant improvement of measurements.

For the future work, we are going to focus on sensor fusion using Kalman filters, in which we also try to use differential evolution or another optimization algorithm.

\section{Acknowledgments}

This work was supported by Internal Grant Agency of Tomas Bata University in Zlin under the project No. IGA/FAI/2018/012. 


\section{References}

[1] Oboe, R. (2001). Use of MEMS based accelerometers in hard disk drives, 2001 IEEE/ASME International Conference on Advanced Intelligent Mechatronics. Proceedings (Cat. No.01TH8556), vol. 2, pp. 1142-1147.

[2] Abramovitch, D. Y. \& Hsu, G. (2015). Mitigating rotational disturbances on a disk drive with mismatched linear accelerometers, 2015 IEEE Conference on Control Applications (CCA), pp. 1473-1478.

[3] Nelson, G. \& Rajamani, R. (2016). Accelerometer Based Acoustic Control: Enabling Auscultation on a Black Hawk Helicopter, IEEE/ASME Trans. Mechatronics, pp. 1-1.

[4] Wei, X. (2016). Autonomous control system for the quadrotor unmanned aerial vehicle, 2016 13th International Conference on Ubiquitous Robots and Ambient Intelligence (URAI), pp. 796-799.

[5] Wu, C.; Mu, Q.; Zhang, Z.; Jin, Y.; Wang, Z. \& Shi, G. (2016). Indoor positioning system based on inertial MEMS sensors: Design and realization, 2016 IEEE International Conference on Cyber Technology in Automation, Control, and Intelligent Systems (CYBER), pp. 370-375.

[6] Xinxi, Z.; Rong, Z.; Meifeng, G.; Gaofeng, C.; Shulai, N. \& Jinglong, L. (2015). The performance impact evaluation on bias of gyro and accelerometer for foot-mounted INS, 2015 12th IEEE International Conference on Electronic Measurement \& Instruments (ICEMI), pp. 1541-1546.

[7] Kuncar, A.; Sysel, M. \& Urbanek, T. (2017). Differential Evolution as Calibration Technique for Three Axis Gyroscope, The 28th DAAAM International Symposium.

[8] Frosio, I.; Pedersini, F. \& Borghese, N. A. (2009). Autocalibration of MEMS Accelerometers, IEEE Trans. Instrum. Meas., vol. 58, no. 6, pp. 2034-2041.

[9] Guo, P.; Qiu, H.; Yang, Y. \& Ren, Z. (2008). The soft iron and hard iron calibration method using extended kalman filter for attitude and heading reference system, 2008 IEEE/ION Position, Location and Navigation Symposium, pp. 1167-1174.

[10] Crassidis, J. L.; Lai, K.-L. \& Harman, R. R. (2005). Real-Time Attitude-Independent Three-Axis Magnetometer Calibration, J. Guid. Control. Dyn., vol. 28, no. 1, pp. 115-120.

[11] Ammann, N.; Derksen, A. \& Heck, C. (2015). A novel magnetometer-accelerometer calibration based on a least squares approach, 2015 International Conference on Unmanned Aircraft Systems (ICUAS), pp. 577-585.

[12] Olsson, F.; Kok, M.; Halvorsen, K. \& Schon, T. B. (2016). Accelerometer calibration using sensor fusion with a gyroscope, 2016 IEEE Statistical Signal Processing Workshop (SSP), pp. 1-5.

[13] Zhang, R.; Hoflinger, F. \& Reind, L. M. (2014). Calibration of an IMU Using 3-D Rotation Platform, IEEE Sens. J., vol. 14 , no. 6 , pp. $1778-1787$.

[14] National Centers of Environmental Information, (2016). [Online]. Available: http://www.ngdc.noaa.gov/geomagweb/?model=igrf\#igrfwmm.

[15] Storn R. \& Price, K. (1995). Differential Evolution - A simple and efficient adaptive scheme for global optimization over continuous spaces. 